\title{
Total synthesis of the bicyclo[6.3.0]undecane-based sesquiterpene ( \pm )-asterisca-3(15),6-diene. Revision of the relative stereochemistry of the natural product
}

\author{
Goverdhan Mehta* and Jayant D. Umarye \\ Department of Organic Chemistry, Indian Institute of Science, Bangalore 560 012, India
}

\begin{abstract}
The first total synthesis of the novel bicyclic sesquiterpene hydrocarbon asterisca-3(15),6-diene is reported. As a consequence, the natural product is shown to possess structure $\mathbf{4}$ with a trans-5,8 ring fusion and not the previously assigned cis-asterisca-3(15),6-diene $\mathbf{3}$.
\end{abstract}

In 1985, a novel 5,8-ring fused sesquiterpene asteriscanolide 1 was isolated from Asteriscus aquaticus $\mathrm{L}$. (family compositae). ${ }^{\text {a }}$ Subsequently, two new natural products $3 \alpha$-hydroxy-6-asteriscene $2^{1 \mathrm{~b}}$ and asterisca3(15), 6-diene $3^{\text {ic }}$ from the essential oil of Lippia integrifolia (Griseb) were added in 1995 and 1999, respectively, to this rare bicyclo[6.3.0]undecane-based family of sesquiterpenes. While the stereostructure of asteriscanolide 1 was secured through a X-ray crystal structure determination, ${ }^{\text {la }}$ those of $\mathbf{2}$ and $\mathbf{3}$ were largely deduced from the analyses of their spectral data. ${ }^{1 \mathrm{~b}, \mathrm{c}}$

The absolute configuration of $\mathbf{2}$ and $\mathbf{3}$ remains unknown. All the three asteriscanes 1-3 were formu- lated as having a cis-fused 5,8-ring junction. Asteriscanolide 1, being the first member of this new skeletal type among sesquiterpenes, has aroused considerable synthetic interest ${ }^{2}$ and four total syntheses ${ }^{3 a-d}$ and several synthetic approaches ${ }^{3 e-g}$ have been reported. However, synthetic endeavours towards $\mathbf{2}$ and $\mathbf{3}$ have not been reported in the literature so far. We describe here the first synthesis of the natural product asterisca3(15),6-diene and demonstrate that the stereostructure of the naturally occurring hydrocarbon needs to be revised to $\mathbf{4}$ with trans-ring fusion.

Our approach to the bicyclo[6.3.0]undecane system was based on the 'carbocyclic ring equivalency' concept. ${ }^{4}$

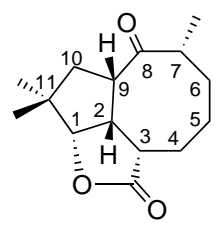

1

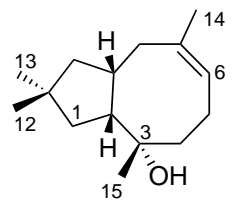

2

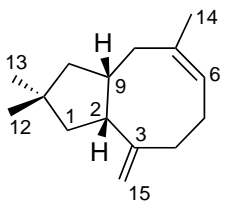

3

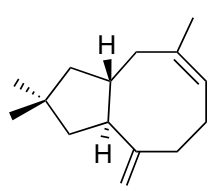

4

"proposed structure" "correct structure"

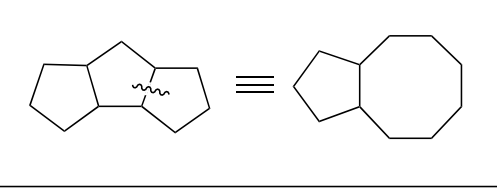

Scheme 1.

Keywords: terpenes; polyquinanes; cyclooctanes; Wittig reaction; stereochemistry.

* Corresponding author. E-mail: gm@orgchem.iisc.ernet.in 
Thus, bicyclo[3.3.0]octane is an eight-membered ring equivalent and tricyclo[6.3.0.0 $\left.0^{2,6}\right]$ undecane (linear triquinane) is the latent form of the bicyclo[6.3.0] undecane system (Scheme 1). In the backdrop of this theme, our synthesis of $\mathbf{3}$ (corresponding to the previously assigned ${ }^{1 \mathrm{c}}$ cis-fused formulation) emanated from the cis,syn,cis-triquinane bis-enone $\mathbf{6}$, readily and quantitatively available from the pentacyclic-caged dione 5 through flash-vacuum pyrolysis (FVP) as described by us many years ago. ${ }^{5}$ Relocation of one of the enone moieties in $\mathbf{6}$ through thermal activation under static conditions led to the bis-enone 7 (Scheme 2). Controlled, selective catalytic hydrogenation to $\mathbf{8}$ and regioselective gem-dimethylation delivered $9 .{ }^{6}$ Chemoselective thioketalisation in 9 gave monothioketal 10, which was subjected to reductive desulfurisation in metal-ammonia milieu to yield a diastereomeric mixture (2:1) of exo-11a and endo-11b alcohols (Scheme 2). The major alcohol 11a was deoxygenated following the Barton protocol ${ }^{7}$ and the resulting tricyclic tetrasubstituted olefin 12 on catalytic ruthenium oxidation afforded the 5,8-fused cis-bicyclic dione $\mathbf{1 3}^{5}$ (Scheme 2).

Wittig olefination of cis-bicyclic dione 13 proceeded regioselectively at the carbonyl group distant from the ring junction and the gem-dimethyl group to furnish keto-olefin 14. ${ }^{6}$ Isomerisation of the exocyclic double bond to the desired endocyclic position in $\mathbf{1 4}$ proved to be difficult due to unwanted transannular cyclisations. ${ }^{8}$ Consequently, the carbonyl group in $\mathbf{1 4}$ was reduced and the resulting hydroxyl compound was protected as a TMS-ether to give 15. Rhodium-mediated olefin isomerisation in 15 led to a mixture of 16a and 16b (4:1) which could be readily separated. TMS-ether deprotection of the required isomer 16a and PDC-oxidation yielded keto-olefin $17^{6}$ (Scheme 3 ). Finally, Wittig olefination of $\mathbf{1 7}$ furnished $\mathbf{3}$, corresponding to the 'assigned structure' of the natural product ${ }^{\text {lc }}$ (Scheme 3).

However, the spectral data $\left({ }^{1} \mathrm{H}\right.$ and $\left.{ }^{13} \mathrm{C} N M R\right){ }^{6}$ of synthetic 3 was distinctly different from that reported for the natural product. ${ }^{1 c}$ Careful scrutiny of the spectral data led us to surmise that the natural product was the trans-isomer $\mathbf{4}$ and we undertook its synthesis.

cis-Bicyclic dione 13 on exposure to base could be readily equilibrated with its trans-isomer $\mathbf{1 8}$ in which the latter was the major product $(4: 1)$. Bicyclic transdione 18, like its cis sibling 13, also underwent a facile regioselective Wittig olefination to yield keto-olefin 19 (Scheme 4). $\mathrm{RhCl}_{3}$-mediated double-bond isomerisation in 19 proceeded without any complications and gave a mixture of readily separable olefinic ketones 20a and 20b (40:60) (Scheme 4). Wittig olefination on the major compound $20 \mathrm{~b}$ proceeded smoothly to furnish the bicyclic hydrocarbon 4 whose spectral characteristics $\left({ }^{1} \mathrm{H}\right.$ and ${ }^{13} \mathrm{C}$ NMR) exactly matched those reported for the natural product. ${ }^{1 \mathrm{c}, 6}$

In short, we have accomplished the total synthesis of the natural product ( \pm )-asterisca-3(15),6-diene and our synthetic efforts necessitates the revision of the earlierassigned structure ${ }^{1 \mathrm{c}}$ of the natural product from cis-3 to trans-4. The synthetic methodology reported here is of general applicability and can be readily adapted for the synthesis of other asteriscane sesquiterpenes.

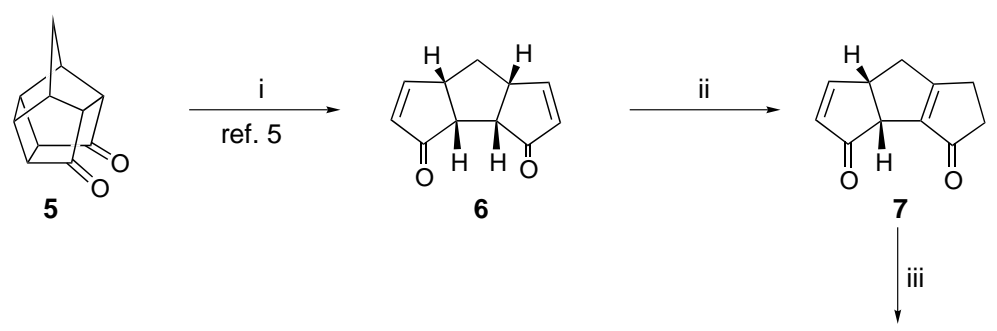

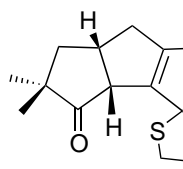

10

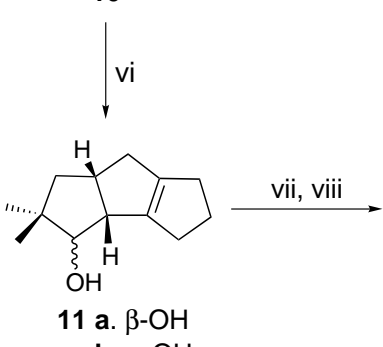

b. $\alpha-\mathrm{OH}$
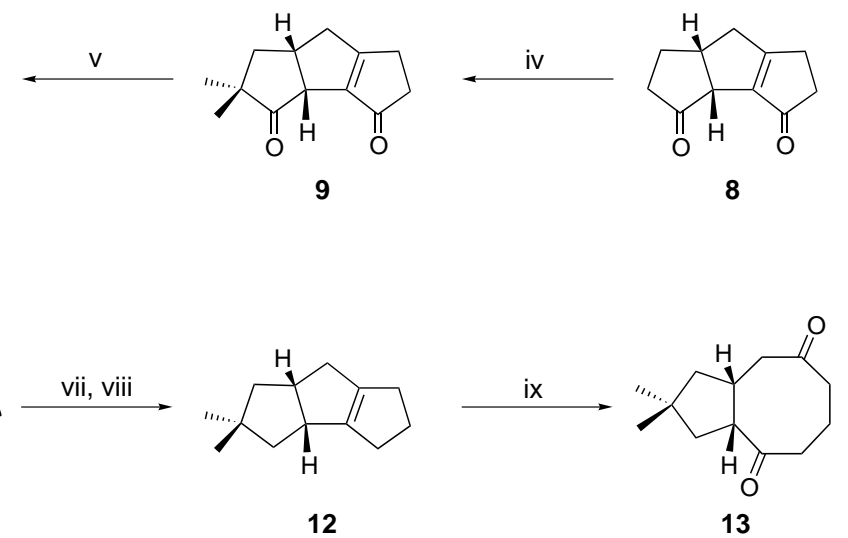

13

Scheme 2. Reagents and conditions: (i) $580^{\circ} \mathrm{C}, \mathrm{FVP}, 0.1$ torr, quantitative; (ii) diphenyl ether, $260{ }^{\circ} \mathrm{C}, 30 \mathrm{~min}, 80 \%$; (iii) $\mathrm{H}_{2}, 10 \%$ $\mathrm{Pd} / \mathrm{C}$, EtOAc, 1 h, 95\%; (iv) $\mathrm{NaH}$ (2 equiv.), THF, MeI (2.5 equiv.), 5-12 h, 35-50\%; (v) ethanedithiol, $\mathrm{BF}_{3}$-etherate, $\mathrm{MeOH}$, 0-10 ${ }^{\circ} \mathrm{C}, 70 \%$; (vi) Na-liq. $\mathrm{NH}_{3}$, THF, $30 \mathrm{~min}, 85 \%$ (2:1 exo:endo epimers); (vii) $\mathrm{NaH}, \mathrm{THF}$, imidazole, $\mathrm{CS}_{2}, \mathrm{MeI}, \mathrm{reflux}, 95 \%$; (viii) $(n-\mathrm{Bu})_{3} \mathrm{SnH}$, benzene, reflux, $2 \mathrm{~h}$; (ix) $\mathrm{RuCl}_{3}, \mathrm{NaIO}_{4}, \mathrm{CCl}_{4}-\mathrm{CH}_{3} \mathrm{CN}-\mathrm{H}_{2} \mathrm{O}, 4 \mathrm{~h}, 70 \%$ after two steps. 


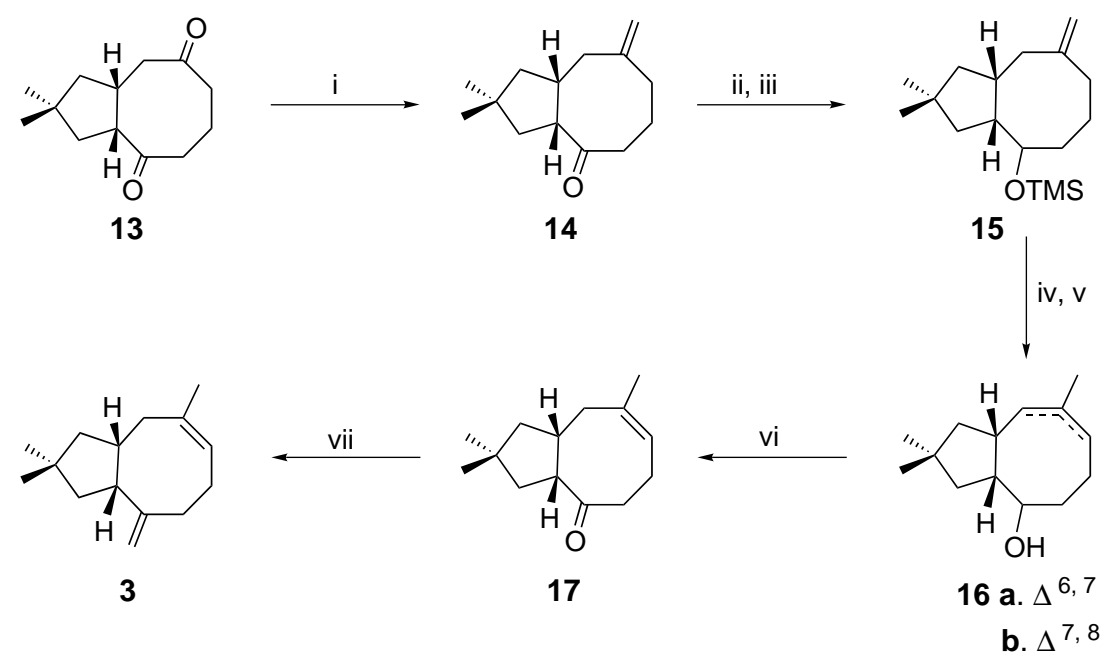

Scheme 3. Reagents and conditions: (i) $\mathrm{MePh}_{3} \mathrm{P}^{+} \mathrm{Br}^{-}, \mathrm{KO}^{t} \mathrm{Bu}$, benzene, $40^{\circ} \mathrm{C}, 30 \mathrm{~min}, 75 \%$; (ii) $\mathrm{NaBH}_{4}, \mathrm{MeOH}^{\mathrm{rt}}, 1 \mathrm{~h}, 75 \%$; (iii) TMS-imidazole, TBAF (cat.), THF, $15 \mathrm{~min}, 95 \%$; (iv) $\mathrm{RhCl}_{3} \cdot 3 \mathrm{H}_{2} \mathrm{O}, \mathrm{NaHCO}_{3}, \mathrm{EtOH}$, reflux, $1 \mathrm{~h}$; (v) $10 \% \mathrm{HCl}, \mathrm{THF}, 0^{\circ} \mathrm{C}, 15 \mathrm{~min}$ (1:4 mixture of 16a and 16b, respectively), 75\% after two steps; (vi) PDC, $4 \AA$ MS powder, DCM, rt, 3 h, 70\%; (vii) $\mathrm{MePh}_{3} \mathrm{P}^{+} \mathrm{Br}^{-}$, $\mathrm{KO}^{t} \mathrm{Bu}$, benzene, $40^{\circ} \mathrm{C}, 30 \mathrm{~min}, 95 \%$.
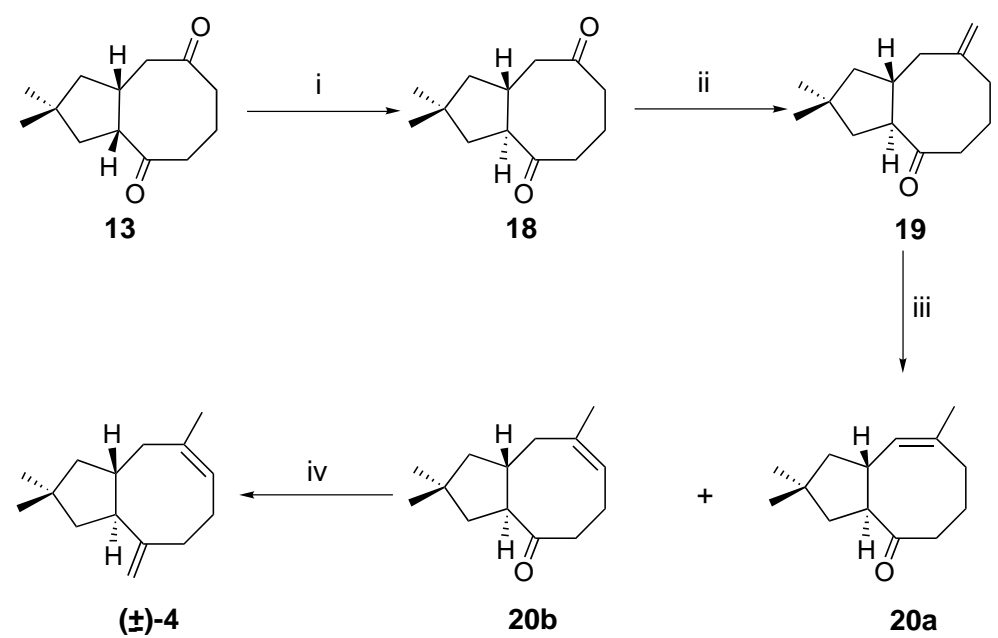

Scheme 4. Reagents and conditions: (i) $\mathrm{KO}^{t} \mathrm{Bu}, \mathrm{THF},{ }^{t} \mathrm{BuOH}, 20 \mathrm{~min}$ (4:1 equilibrium mixture of 18 and 13 , respectively), quantitative; (ii) $\mathrm{MePh}_{3} \mathrm{P}^{+} \mathrm{Br}^{-}, \mathrm{KO}^{t} \mathrm{Bu}$, benzene, $40^{\circ} \mathrm{C}, 30 \mathrm{~min}, 75 \%$; (iii) $\mathrm{RhCl}_{3} \cdot 3 \mathrm{H}_{2} \mathrm{O}, \mathrm{NaHCO}_{3}, \mathrm{EtOH}, \Delta, 1 \mathrm{~h}(2: 3 \mathrm{mixture}$ of $20 \mathrm{a}$ and 20b, respectively), 90\%; (iv) $\mathrm{MePh}_{3} \mathrm{P}^{+} \mathrm{Br}^{-}, \mathrm{KO}^{t} \mathrm{Bu}$, benzene, $40^{\circ} \mathrm{C}, 30 \mathrm{~min}, 95 \%$.

\section{Acknowledgements}

One of us (J.D.U.) thanks the CSIR for the award of a Research Fellowship. We thank SIF at IISc for help with the high-field NMR data.

\section{References}

1. (a) San Feliciano, A.; Barrero, A. F.; Medarde, M.; Miguel de Corral, J. M.; Aramburu, A. Tetrahedron Lett. 1985, 26, 2369; (b) Catalan, C. A. N.; de Lampasona, M. E. P.; Cerda-Garcia-Rojas, C. M.; Joseph-Nathan, P. J. Nat. Prod. 1995, 58, 1713; (c) Fricke, C.; Hardt, I. H.; Konig, W. A.; Joulain, D.; Zygadlo, J. A.; Guzman, C. A. J. Nat. Prod. 1999, 62, 694.
2. For a recent review, see: Mehta, G.; Singh, V. K. Chem. Rev. 1999, 99, 881.

3. (a) Wender, P. A.; Ihle, N. C.; Correia, C. R. D. J. Am. Chem. Soc. 1988, 110, 5904; (b) Krafft, M. E.; Cheung, Y.-Y.; Juliano-Capucao, C. A. Synthesis 2000, 1020; (c) Paquette, L. A.; Tae, J.; Arrington, M. P.; Sadoun, A. H. J. Am. Chem. Soc. 2000, 122, 2742; (d) Limanto, J.; Snapper, M. L. J. Am. Chem. Soc. 2000, 122, 8071; (e) Lange, G. L.; Organ, M. G. J. Org. Chem. 1996, 61, 5358; (f) Booker-Milburn, K. I.; Cowell, J. K.; Harris, L. J. Tetrahedron 1997, 53, 12319; (g) Sarkar, T. K.; Gangopadhyay, P.; Ghorai, B. K.; Nandi, S. K.; Fang, J.-M. Tetrahedron Lett. 1998, 39, 8365.

4. For related examples, see: (a) Mehta, G.; Murty, A. N. $J$. Org. Chem. 1990, 55, 3568; (b) Mehta, G.; Krishnamurthy, N. J. Chem. Soc., Chem. Commun. 1986, 1319; (c) Piers, E.; Skupinska, K. A.; Wallace, D. J. Synlett 1999, 1867. 
5. (a) Mehta, G.; Reddy, A. V.; Srikrishna, A. Tetrahedron Lett. 1979, 4863; (b) Mehta, G.; Srikrishna, A.; Reddy, A. V.; Nair, M. S. Tetrahedron 1981, 37, 4543.

6. All new compounds reported here were racemic and characterised on the basis of spectroscopic data (IR, ${ }^{1} \mathrm{H}$ and ${ }^{13} \mathrm{C}$ NMR, mass) and elemental analyses. Selected spectral data: Compound 13: ${ }^{1} \mathrm{H}$ NMR (300 $\left.\mathrm{MHz}, \mathrm{CDCl}_{3}\right): \delta 3.34$ $(\mathrm{dd}, J=16.5,7.8 \mathrm{~Hz}, 1 \mathrm{H}), 2.93-2.83(\mathrm{~m}, 1 \mathrm{H}), 2.63-2.4(\mathrm{~m}$, $5 \mathrm{H}), 2.24(\mathrm{dd}, J=13.8,3.6 \mathrm{~Hz}, 1 \mathrm{H}), 2.17-2.10(\mathrm{~m}, 2 \mathrm{H})$, $1.93(\mathrm{dd}, J=13.2,7.5 \mathrm{~Hz}, 1 \mathrm{H}), 1.61(\mathrm{dd}, J=12.6,6.3 \mathrm{~Hz}$, $1 \mathrm{H}), 1.48(\mathrm{dd}, J=13.5,7.5 \mathrm{~Hz}, 1 \mathrm{H}), 1.33(\mathrm{dd}, J=12.3,10$ $\mathrm{Hz}, 1 \mathrm{H}), 1.14$ (s, 3H), 0.99 (s, 3H); ${ }^{13} \mathrm{C}$ NMR $(75 \mathrm{MHz}$, $\left.\mathrm{CDCl}_{3}\right): \delta$ 215.0, 213.5, 52.4, 48.0, 44.6, 44.2, 43.6, 42.0, 40.6, 37.6, 29.4, 29.3, 22.5. Compound 18: ${ }^{1} \mathrm{H}$ NMR (300 $\left.\mathrm{MHz}, \mathrm{CDCl}_{3}\right): \delta 2.9(\mathrm{dt}, J=10.8,7.8 \mathrm{~Hz}, 1 \mathrm{H}), 2.60-2.20$ (m, 8H), 2.10-2.03 (m, 1H), 1.88-1.80 (m, 2H), 1.59 (dd, $J=12.9,7.2 \mathrm{~Hz}, 1 \mathrm{H}), 1.30$ (dd as t, $J=12.1 \mathrm{~Hz}, 1 \mathrm{H}), 1.10$ (s, 3H), $1.06(\mathrm{~s}, 3 \mathrm{H}) ;{ }^{13} \mathrm{C} \mathrm{NMR}\left(75 \mathrm{MHz}, \mathrm{CDCl}_{3}\right): \delta 213.7$, 211.8, 56.4, 49.6, 46.9, 44.5, 43.4, 42.8, 42.6, 36.6, 31.4, 30.9, 21.6. Compound 3: ${ }^{1} \mathrm{H}$ NMR (300 MHz, $\left.\mathrm{CDCl}_{3}\right): \delta$ $5.38(\mathrm{t}, J=7.2 \mathrm{~Hz}, 1 \mathrm{H}), 4.92(\mathrm{~s}, 1 \mathrm{H}), 4.77(\mathrm{~s}, 1 \mathrm{H}), 2.66(\mathrm{q}$, $J=7.5 \mathrm{~Hz}, 1 \mathrm{H}$ ), 2.5-1.9 (series of m, 6H), 1.7 (s, 3H), 1.68 $(\mathrm{m}, 1 \mathrm{H}), 1.62(\mathrm{dd}, J=15.0,6.6 \mathrm{~Hz}, 1 \mathrm{H}), 1.56(\mathrm{~m}, 1 \mathrm{H})$, 1.45-1.27 (series of $\mathrm{d}, 2 \mathrm{H}), 1.11(\mathrm{~s}, 3 \mathrm{H}), 1.02(\mathrm{~s}, 3 \mathrm{H}) ;{ }^{13} \mathrm{C}$
NMR (75 MHz, $\left.\mathrm{CDCl}_{3}\right): \delta 154.6,138.5,123.1,112.4,47.6$, 47.3, 47.2, 41.9, 40.9, 37.0, 36.2, 30.6, 30.0, 27.8, 24.3. Compound 4: ${ }^{1} \mathrm{H}$ NMR $\left(300 \mathrm{MHz}, \mathrm{CDCl}_{3}\right): \delta 5.20(\mathrm{~m}$, $1 \mathrm{H}), 4.80(\mathrm{~s}, 1 \mathrm{H}), 4.66(\mathrm{~s}, 1 \mathrm{H}), 2.43(\mathrm{~m}, 1 \mathrm{H}), 2.32-2.26(\mathrm{~m}$, $1 \mathrm{H}), 2.22-2.05(\mathrm{~m}, 3 \mathrm{H}), 1.98-1.94(\mathrm{~m}, 1 \mathrm{H}), 1.83(\mathrm{dd}$, $J=13.2,2.4 \mathrm{~Hz}, 1 \mathrm{H}), 1.68(\mathrm{~m}, 1 \mathrm{H}), 1.68(\mathrm{~s}, 3 \mathrm{H}), 1.59$ (d, $J=9.6 \mathrm{~Hz}, 2 \mathrm{H}), 1.56-1.48(\mathrm{~m}, 1 \mathrm{H}), 1.17(\mathrm{dd}$ as $\mathrm{t}, J=11.7$ $\mathrm{Hz}, 1 \mathrm{H}), 1.08$ (s, 3H), 1.02 (s, 3H) ${ }^{13} \mathrm{C}$ NMR $(75 \mathrm{MHz}$, $\left.\mathrm{CDCl}_{3}\right): \delta 151.9,137.1,123.4,109.3,50.0,49.6,49.4,47.7$, 39.4, 37.4, 35.0, 31.8, 31.7, 25.1, 24.5.

7. Barton, D. H. R.; McCombie, S. W. J. Chem. Soc., Perkin Trans. 1 1975, 1574.

8. On exposure to $\mathrm{RhCl}_{3} \cdot 3 \mathrm{H}_{2} \mathrm{O}$, the keto-olefin 14 exclusively furnished the transannularly cyclised product (i), which proved quite unserviceable for further elaboration towards the natural product.

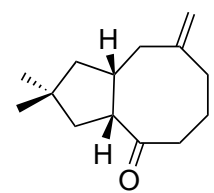

14

\section{$\mathrm{RhCl}_{3} .3 \mathrm{H}_{2} \mathrm{O}$ \\ $\mathrm{EtOH}, \Delta, 1 \mathrm{~h}$}

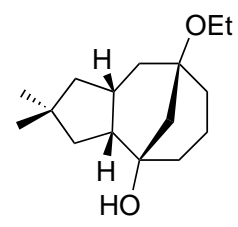

(i) 